\title{
Focusing of spatially inhomogeneous partially coherent, partially polarized electromagnetic fields
}

\author{
Matthew R. Foreman and Peter Török* \\ Blackett Laboratory, Department of Physics, Imperial College London, Prince Consort Road, London SW7 2BZ, UK \\ *Corresponding author: peter.torok@imperial.ac.uk
}

Received July 17, 2009; revised September 21, 2009; accepted September 22, 2009;

posted September 25, 2009 (Doc. ID 114459); published October 30, 2009

\begin{abstract}
We report a general framework capable of describing the focusing of electromagnetic waves with spatially varying coherence and polarization properties in optical systems of arbitrary numerical aperture and Fresnel number. We also investigate the reduction of the dimensionality of the requisite integrals by use of a coherent mode expansion. We find that coherent mode expansions treating each component of the electric field vector individually are unsuitable for describing focusing systems because of the inter-component mixing that can occur in high numerical aperture systems. In addition, we show that the assumption of harmonic angular dependence allows the azimuthal integration to be performed analytically, providing further simplification of the analysis. We also find that the effective degree of spectral coherence of an electromagnetic beam is unchanged upon focusing. Finally, as an illustration of the developed framework, we calculate the transverse and axial focal distributions for a partially coherent source formed by incoherent superposition of radially and azimuthally polarized Laguerre-Gauss modes. () 2009 Optical Society of America
\end{abstract}

OCIS codes: $030.1640,260.5430$.

\section{INTRODUCTION}

Focusing in optical systems has been researched in earnest for myriad different scenarios. Focusing of coherent light under a scalar approximation has been well understood for many years (see, for example, [1]), while even as early as 1919 focusing of coherent, fully polarized electromagnetic waves could be described by what is now known as the Debye-Wolf diffraction integral [2-5]. In more recent years attention has slowly turned toward focusing of partially coherent light in both scalar [6-9] and vectorial $[10,11]$ regimes due to its potential use in lithography, laser fusion, and microscopy [12-15]. Consideration of the full electromagnetic problem has, however, been limited to homogeneous (partial) polarization across the pupil of the focusing lens. A full and general treatment of the focusing of inhomogeneous, partially coherent, partially polarized waves is therefore lacking, an omission that is addressed in this article.

In Section 2 we first introduce the scaled Debye-Wolf diffraction integral, which describes focusing in systems of arbitrary numerical aperture and Fresnel number. The scaled Debye-Wolf integral is then applied to focusing of arbitrary partially coherent, partially polarized light in Section 3. The general result derived in Section 3 requires evaluation of a fourfold integral; however, by using a coherent mode expansion the dimensionality of the problem can be reduced, as discussed in Section 4 for both scalarand vector-based mode expansions. Further simplifications can be made when the coherent modes possess a harmonic angular dependence, since the azimuthal integration can be evaluated analytically, as shall be demonstrated in Section 5. Finally, in Section 6 we give numeri- cal results for two examples of practical importance, namely, radially and azimuthally polarized beams formed from Laguerre-Gauss modes.

\section{SCALED DEBYE-WOLF DIFFRACTION INTEGRAL}

The scaled Debye-Wolf diffraction integral can be used to describe focusing of quasi-monochromatic, coherent light in optical systems with an arbitrary, albeit finite, Fresnel number and arbitrary numerical aperture and is given by [16]

$$
\begin{aligned}
\mathbf{E}(\mathbf{r})= & -\frac{i f^{2} \exp \left(i k \Phi_{0}\right)}{\lambda(f+z)} \iint_{s_{x}^{2}+s_{y}^{2} \leqslant 1} \mathbf{e}\left(s_{x}, s_{y}\right) \\
& \times \exp (i k \mathbf{s} \cdot \mathbf{P}) \frac{\mathrm{d} s_{x} \mathrm{~d} s_{y}}{s_{z}},
\end{aligned}
$$

where $\mathbf{P}=(R \cos \varphi, R \sin \varphi, Z)$ represents the position vector $\mathbf{r}=(\rho \cos \varphi, \rho \sin \varphi, z)$ of a point of observation in the focal region, in a transformed coordinate system with

$$
\begin{aligned}
& R=\frac{f}{f+z} \rho, \\
& Z=\frac{f}{f+z} z .
\end{aligned}
$$

$\mathbf{s}=\left(s_{x}, s_{y}, s_{z}\right)=(\sin \theta \cos \phi, \sin \theta \sin \phi, \cos \theta)$ is a unit vector describing the direction of a ray (see Fig. 1), $f$ is the focal length of the lens, $k=2 \pi / \lambda=\omega / c$ is the wavenumber of 


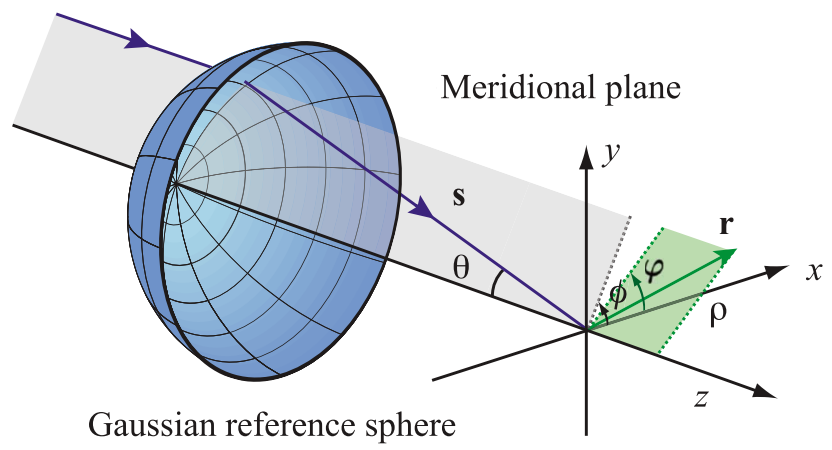

Fig. 1. (Color online) Coordinate system and geometry of the scaled Debye-Wolf diffraction integral.

light of wavelength $\lambda$ and frequency $\omega$,

$$
\Phi_{0}=f+z+\frac{\rho^{2}-2 f z}{2(f+z)},
$$

and $\mathbf{e}\left(s_{x}, s_{y}\right)$ describes the field distribution on the Gaussian reference sphere located in the exit pupil of the system centered on the geometrical focus of the lens. Noting that an element of solid angle over the reference sphere is given by

$$
\frac{\mathrm{d} s_{x} \mathrm{~d} s_{y}}{s_{z}}=\sin \theta \mathrm{d} \theta \mathrm{d} \phi,
$$

the scaled Debye-Wolf integral can be rewritten as

$$
\begin{aligned}
\mathbf{E}(\rho, \varphi, z)= & -\frac{i f^{2} \exp \left(i k \Phi_{0}\right)}{\lambda(f+z)} \int_{0}^{2 \pi} \int_{0}^{\alpha} \mathbf{e}(\theta, \phi) \\
& \times \exp [i k R \sin \theta \cos (\phi-\varphi)] e^{i k Z \cos \theta} \sin \theta \mathrm{d} \theta \mathrm{d} \phi,
\end{aligned}
$$

where $\alpha$ is the semi-angle of convergence of the lens. For systems of large Fresnel numbers (whereby $R \approx \rho$ and $Z$ $\approx z$ ) the scaled Debye-Wolf integral reduces to the more familiar Debye-Wolf integral [4,5]

$$
\begin{aligned}
\mathbf{E}(\rho, \varphi, z)= & -\frac{\text { if }}{\lambda} \int_{0}^{2 \pi} \int_{0}^{\alpha} \mathbf{e}(\theta, \phi) \\
& \times \exp [i k \rho \sin \theta \cos (\phi-\varphi)] e^{i k z \cos \theta} \sin \theta \mathrm{d} \theta \mathrm{d} \phi .
\end{aligned}
$$

\section{FOCUSING OF PARTIALLY COHERENT, PARTIALLY POLARIZED LIGHT}

Partially coherent light is generally described by using its second-order statistical properties. For example, when considering scalar fields, one may use either the mutual coherency function or the cross-spectral density function [17]. For electromagnetic fields the natural treatment is a matrix formulation, with the second-order properties in the space-time domain being described by a mutual coherency matrix, first introduced by Wiener [18,19], although also independently later introduced by Wolf [20]. More recently an alternative space-frequency domain formalism has been developed. The correlation matrix of interest is then often referred to as the cross-spectral density matrix (CSDM), which is itself a Fourier transform of a mutual coherency matrix [21]. It is worth noting that not only does the CSDM describe spatial coherence properties of a stochastic field (i.e., correlations between the field at two different points in space), but it also describes partial polarization as encapsulated in the off-diagonal elements, which measure the correlation between orthogonal field components. Consequently the work that follows applies to focusing of stochastic electromagnetic fields with arbitrary coherence and polarization properties.

Throughout this work we shall denote the CSDM in the focal region of a lens as

$$
\mathbb{W}\left(\mathbf{r}_{1}, \mathbf{r}_{2}, \omega\right) \equiv\left\langle\mathbf{E}\left(\mathbf{r}_{1}, \omega\right) \mathbf{E}^{\dagger}\left(\mathbf{r}_{2}, \omega\right)\right\rangle
$$

where ${ }^{\dagger}$ denotes the Hermitian adjoint operator and $\langle\cdots\rangle$ denotes the ensemble average over many monochromatic statistical realisations, $\mathbf{E}(\mathbf{r}, \omega)$, of the electromagnetic field. For brevity the frequency dependence of all quantities will be dropped for the remainder of this article. The CSDM over other domains in the optical system, e.g., over the reference sphere, can be expressed in a similar manner. Table 1 gives a summary of the notation used for the CSDM and other related quantities at different points in the focusing system.

Given Eq. (8) and the scaled Debye-Wolf integral [Eq. (6)] it is a simple matter to determine the CSDM for light focused by a lens. By substitution we have

$$
\begin{aligned}
\mathbb{W}\left(\mathbf{r}_{1}, \mathbf{r}_{2}\right)= & K_{1} K_{2}^{*} \int_{0}^{2 \pi} \int_{0}^{2 \pi} \int_{0}^{\alpha} \int_{0}^{\alpha}\left\langle\mathbf{e}\left(\theta_{1}, \phi_{1}\right) \mathbf{e}^{\dagger}\left(\theta_{2}, \phi_{2}\right)\right\rangle \\
& \times \exp \left[i k \Delta_{12}\right] \exp \left[i k\left(Z_{1} \cos \theta_{1}-Z_{2} \cos \theta_{2}\right)\right] \\
& \times \sin \theta_{1} \sin \theta_{2} \mathrm{~d} \theta_{1} \mathrm{~d} \theta_{2} \mathrm{~d} \phi_{1} \mathrm{~d} \phi_{2}
\end{aligned}
$$

Table 1. Summary of Notation

\begin{tabular}{lccc}
\hline & Gaussian Reference & & \\
& Sphere & Back Focal Plane & Focal Region \\
\hline Coordinates & $\{\theta, \phi\}$ & $\{\theta, \phi\}$ & $\mathbf{r}=\{\rho, \varphi, z\}$ \\
Electric field vector & $\mathbf{e}(\theta, \phi)$ & $\mathbf{E}(\theta, \phi)$ & $\mathbb{E}(\mathbf{r})$ \\
Cross-spectral density matrix & $\mathrm{w}\left(\theta_{1}, \phi_{1}, \theta_{2}, \phi_{2}\right)$ & $\widetilde{\mathbb{W}}\left(\theta_{1}, \phi_{1}, \theta_{2}, \phi_{2}\right)$ & $\mathbb{W}\left(\mathbf{r}_{1}, \mathbf{r}_{2}\right)$ \\
Scalar-based coherent mode & $\psi_{n}^{(i)}(\theta, \phi)$ & $\widetilde{\psi}_{n}^{(i)}(\theta, \phi)$ & $C_{n}^{(i)}(\mathbf{r})$ \\
Vector-based coherent mode & $\boldsymbol{\Psi}_{n}(\theta, \phi)$ & $\widetilde{\Psi}_{n}(\theta, \phi)$ & $\hat{\mathbf{C}}_{n}(\mathbf{r})$ \\
\hline
\end{tabular}


where $\quad K_{l}=-\left[\left(i k f^{2}\right) / 2 \pi\left(f+z_{l}\right)\right] \exp \left(i k \Phi_{0}\right) \quad$ and $\quad \Delta_{12}$ $=R_{1} \sin \theta_{1} \cos \left(\phi_{1}-\varphi_{1}\right)-R_{2} \sin \theta_{2} \cos \left(\phi_{2}-\varphi_{2}\right)$. Knowledge of the CSDM in a single transverse plane is sufficient to calculate the CSDM on any transverse plane in the focal region via, for example, the Wolf equations [21]. Henceforth we shall thus make the simplifying assumption that $Z_{1}=Z_{2}=Z$; i.e., we restrict attention to a single plane in the focal region. Consequently we also have $K_{1}=K_{2}=K$.

Finally, defining the CSDM on the Gaussian reference sphere in a way analogous to Eq. (8) such that $\mathrm{w}\left(\theta_{1}, \phi_{1}, \theta_{2}, \phi_{2}\right)=\left\langle\mathbf{e}\left(\theta_{1}, \phi_{1}\right) \mathbf{e}^{\dagger}\left(\theta_{2}, \phi_{2}\right)\right\rangle$ gives

$$
\begin{aligned}
\mathbb{W}\left(\mathbf{r}_{1}, \mathbf{r}_{2}\right)= & |K|^{2} \int_{0}^{2 \pi} \int_{0}^{2 \pi} \int_{0}^{\alpha} \int_{0}^{\alpha} \mathrm{w}\left(\theta_{1}, \phi_{1}, \theta_{2}, \phi_{2}\right) \\
& \times \exp \left[i k \Delta_{12}\right] \exp \left[i k Z\left(\cos \theta_{1}-\cos \theta_{2}\right)\right] \\
& \times \sin \theta_{1} \sin \theta_{2} \mathrm{~d} \theta_{1} \mathrm{~d} \theta_{2} \mathrm{~d} \phi_{1} \mathrm{~d} \phi_{2} .
\end{aligned}
$$

In some applications it may be more useful to define the focused CSDM in terms of the CSDM in the back focal plane of the lens, which we denote $\widetilde{\mathbb{W}}\left(\theta_{1}, \phi_{1}, \theta_{2}, \phi_{2}\right)$ $=\left\langle\widetilde{\mathbf{E}}\left(\theta_{1}, \phi_{1}\right) \widetilde{\mathbf{E}}^{\dagger}\left(\theta_{2}, \phi_{2}\right)\right\rangle$. Since the exit pupil of the focusing lens is located at infinity when viewed from the second principal focal plane, it is legitimate to use an infinite boundary condition to relate the electric field vectors on the reference sphere and in the back focal plane and hence also the associated CSDMs. An infinite boundary condition is equivalent to a geometrical $(\lambda \rightarrow 0)$ boundary condition, and hence it is possible to use the generalized Jones matrix formalism $[22,23]$ to give (assuming an ideal lens and neglecting skew rays)

$$
\mathbf{e}(\theta, \phi)=a(\theta) \mathbb{R}^{-1}(\phi) \cdot \mathbb{L}(\theta) \cdot \mathbb{R}(\phi) \cdot \widetilde{\mathbf{E}}(\theta, \phi)=\mathbb{P}(\theta, \phi) \cdot \widetilde{\mathbf{E}}(\theta, \phi),
$$

where $\mathbb{P}(\theta, \phi)=a(\theta) \mathbb{R}^{-1}(\phi) \cdot \mathbb{L}(\theta) \cdot \mathbb{R}(\phi)$,

$$
\mathbb{R}(\phi)=\left(\begin{array}{ccc}
\cos \phi & \sin \phi & 0 \\
-\sin \phi & \cos \phi & 0 \\
0 & 0 & 1
\end{array}\right)
$$

decomposes the field into $s$ and $p$ field components that lie perpendicular and parallel to the meridional plane, respectively, (see Fig. 1), while

$$
\mathbb{L}(\theta)=\left(\begin{array}{ccc}
\cos \theta & 0 & \sin \theta \\
0 & 1 & 0 \\
-\sin \theta & 0 & \cos \theta
\end{array}\right)
$$

describes the deflection of a ray by the lens. The scalar factor $a(\theta)$ is an apodization factor that ensures that energy is conserved when projecting from the reference sphere to a plane. For example $a(\theta)=\sqrt{\cos \theta}$ or $a(\theta)=1$ if the lens satisfies the sine or Herschel condition respectively [24]. Hence

$$
\mathrm{w}\left(\theta_{1}, \phi_{1}, \theta_{2}, \phi_{2}\right)=\mathbb{P}\left(\theta_{1}, \phi_{1}\right) \cdot \widetilde{\mathbb{W}}\left(\theta_{1}, \phi_{1}, \theta_{2}, \phi_{2}\right) \cdot \mathbb{P}^{\dagger}\left(\theta_{2}, \phi_{2}\right) \text {. }
$$

Substituting Eq. (14) in Eq. (10) yields

$$
\begin{aligned}
\mathbb{W}\left(\mathbf{r}_{1}, \mathbf{r}_{2}\right)= & |K|^{2} \int_{0}^{2 \pi} \int_{0}^{2 \pi} \int_{0}^{\alpha} \int_{0}^{\alpha} \exp \left[i k \Delta_{12}\right] \\
& \times \mathbb{P}\left(\theta_{1}, \phi_{1}\right) \cdot \widetilde{\mathbb{W}}\left(\theta_{1}, \phi_{1}, \theta_{2}, \phi_{2}\right) \cdot \mathbb{P}^{\dagger}\left(\theta_{2}, \phi_{2}\right) \\
& \times \exp \left[i k Z\left(\cos \theta_{1}-\cos \theta_{2}\right)\right] \\
& \times \sin \theta_{1} \sin \theta_{2} \mathrm{~d} \theta_{1} \mathrm{~d} \theta_{2} \mathrm{~d} \phi_{1} \mathrm{~d} \phi_{2} .
\end{aligned}
$$

Equations (10) and (15) are the key results for this paper. In what follows we consider various cases under which the integrals simplify from the fourfold integrals given to separable twofold integrals (Section 4) or, even under certain symmetry assumptions, single integrals (Section 5).

\section{COHERENT MODE REPRESENTATIONS}

Scalar coherent mode expansions in optical coherence theory (see e.g., [21] for a fuller discussion) were perhaps first pioneered by Wolf [25] but have seen fervent use by other authors, e.g., [12,26,27]. It should, however, be noted that all such theories derive from Karhunen-Loève theory $[28,29]$ which has been employed in statistics since the 1940s. Karhunen-Loève theory tells us that given a (Hermitian, nonnegative definite, square integrable) scalar correlation function over a closed domain $D$, such as the cross-spectral density function $W\left(\mathbf{r}_{1}, \mathbf{r}_{2}, \omega\right)$, it is possible to expand it in terms of an infinite, orthonormal set of coherent modes, $\psi_{n}(\mathbf{r}, \omega)$, viz.

$$
W\left(\mathbf{r}_{1}, \mathbf{r}_{2}\right)=\sum_{n=0}^{\infty} \lambda_{n} \psi_{n}^{*}\left(\mathbf{r}_{1}\right) \psi_{n}\left(\mathbf{r}_{2}\right),
$$

where the coherent modes and associated expansion coefficients $\lambda_{n}(\omega)$ are found by solution of the Fredholm integral equation:

$$
\int_{D} W\left(\mathbf{r}_{1}, \mathbf{r}_{2}\right) \psi_{n}\left(\mathbf{r}_{1}\right) \mathrm{d} \mathbf{r}_{1}=\lambda_{n} \psi_{n}\left(\mathbf{r}_{2}\right) .
$$

Extension of existing scalar results to a treatment of the full electromagnetic problem is, however, more controversial, with two opposing schools of thought debating the appropriate form of coherent mode expansions for (twoand) three-dimensional fields. In what follows we shall consider both of the alternative formalisms in turn and shall denote them the scalar- and vector-based formalisms.

The first, scalar-based, interpretation applies the scalar formulation described above to each field component individually, hence requiring the solution of (two) three uncoupled Fredholm integral equations of the form of Eq. (17). Accordingly, the individual elements of a general CSDM are expressed in the form [21]

$$
W_{i j}\left(\mathbf{r}_{1}, \mathbf{r}_{2}\right)= \begin{cases}\sum_{n=0}^{\infty} \lambda_{n}^{(i)} \psi_{n}^{(i) *}\left(\mathbf{r}_{1}\right) \psi_{n}^{(i)}\left(\mathbf{r}_{2}\right) & \text { for } i=j, \\ \sum_{n=0}^{\infty} \sum_{m=0}^{\infty} \Lambda_{n m}^{(i j)} \psi_{n}^{(i) *}\left(\mathbf{r}_{1}\right) \psi_{m}^{(j)}\left(\mathbf{r}_{2}\right) & \text { for } i \neq j,\end{cases}
$$

where $W_{i j}\left(\mathbf{r}_{1}, \mathbf{r}_{2}\right)$ is the $(i, j)$ th element of $\mathbb{W}\left(\mathbf{r}_{1}, \mathbf{r}_{2}\right)$ and the expansion coefficients for off-diagonal terms, $\Lambda_{n m}^{(i j)}$, are found according to the integral 


$$
\Lambda_{n m}^{(i j)}=\int_{D} \int_{D} \psi_{n}^{(i)}\left(\mathbf{r}_{1}\right) W_{i j}\left(\mathbf{r}_{1}, \mathbf{r}_{2}\right) \psi_{m}^{(j) *}\left(\mathbf{r}_{2}\right) \mathrm{d} \mathbf{r}_{1} \mathrm{~d} \mathbf{r}_{2} .
$$

Alternatively, the vector-based formalism solves the Fredholm integral equation with matrix-valued kernel

$$
\int_{D} \mathbb{W}\left(\mathbf{r}_{1}, \mathbf{r}_{2}\right) \Psi_{n}\left(\mathbf{r}_{1}\right) \mathrm{d} \mathbf{r}_{1}=\lambda_{n} \boldsymbol{\Psi}_{n}\left(\mathbf{r}_{2}\right)
$$

to find vectorial coherent modes [30,31], such that

$$
\mathbb{W}\left(\mathbf{r}_{1}, \mathbf{r}_{2}\right)=\sum_{n=0}^{\infty} \lambda_{n} \boldsymbol{\Psi}_{n}\left(\mathbf{r}_{1}\right) \boldsymbol{\Psi}_{n}^{\dagger}\left(\mathbf{r}_{2}\right) .
$$

Although this approach is more mathematically involved, since it requires the solution of (two) three coupled scalar Fredholm integral equations, it does express the offdiagonal elements more concisely.

Motivated by the analytic advantages frequently afforded by use of coherent mode expansions, we now use them in our description of focusing of partially polarized, partially coherent light. Furthermore, we shall consider expansions of the CSDMs w $\left(\theta_{1}, \phi_{1}, \theta_{2}, \phi_{2}\right)$ on the reference sphere and $\widetilde{\mathbb{W}}\left(\theta_{1}, \phi_{1}, \theta_{2}, \phi_{2}\right)$ in the back focal plane.

Consider first the scalar-based expansion of w $\left(\theta_{1}, \phi_{1}, \theta_{2}, \phi_{2}\right)$. Using Eq. (10) we immediately have

$$
\begin{aligned}
W_{i j}\left(\mathbf{r}_{1}, \mathbf{r}_{2}\right)= & |K|^{2} \sum_{n=0}^{\infty} \lambda_{n}^{(i)} \\
& \times \int_{0}^{2 \pi} \int_{0}^{2 \pi} \int_{0}^{\alpha} \int_{0}^{\alpha} \psi_{n}^{(i) *}\left(\theta_{1}, \phi_{1}\right) \psi_{n}^{(i)}\left(\theta_{2}, \phi_{2}\right) \\
& \times \exp \left[i k \Delta_{12}\right] \exp \left[i k Z\left(\cos \theta_{1}-\cos \theta_{2}\right)\right] \\
& \times \sin \theta_{1} \sin \theta_{2} \mathrm{~d} \theta_{1} \mathrm{~d} \theta_{2} \mathrm{~d} \phi_{1} \mathrm{~d} \phi_{2} .
\end{aligned}
$$

for $i=j$ and

$$
\begin{aligned}
W_{i j}\left(\mathbf{r}_{1}, \mathbf{r}_{2}\right)= & |K|^{2} \sum_{n=0}^{\infty} \sum_{m=0}^{\infty} \Lambda_{n m}^{(i j)} \\
& \times \int_{0}^{2 \pi} \int_{0}^{2 \pi} \int_{0}^{\alpha} \int_{0}^{\alpha} \psi_{n}^{(i) *}\left(\theta_{1}, \phi_{1}\right) \psi_{m}^{(j)}\left(\theta_{2}, \phi_{2}\right) \\
& \times \exp \left[i k \Delta_{12}\right] \exp \left[i k Z\left(\cos \theta_{1}-\cos \theta_{2}\right)\right] \\
& \times \sin \theta_{1} \sin \theta_{2} \mathrm{~d} \theta_{1} \mathrm{~d} \theta_{2} \mathrm{~d} \phi_{1} \mathrm{~d} \phi_{2} .
\end{aligned}
$$

for $i \neq j$. Letting

$$
\begin{aligned}
C_{n}^{(i)}\left(\mathbf{r}_{l}\right)= & K \int_{0}^{2 \pi} \int_{0}^{\alpha} \psi_{n}^{(i) *}\left(\theta_{l}, \phi_{l}\right) \exp \left[i k R_{l} \sin \theta_{l} \cos \left(\phi_{l}-\varphi_{l}\right)\right] \\
& \times e^{i k Z \cos \theta_{l}} \sin \theta_{l} \mathrm{~d} \theta_{l} \mathrm{~d} \phi_{l}
\end{aligned}
$$

gives

$$
W_{i j}\left(\mathbf{r}_{1}, \mathbf{r}_{2}\right)= \begin{cases}\sum_{n=0}^{\infty} \lambda_{n}^{(i)} C_{n}^{(i)^{*}}\left(\mathbf{r}_{1}\right) C_{n}^{(i)}\left(\mathbf{r}_{2}\right) & \text { for } i=j, \\ \sum_{n=0}^{\infty} \sum_{m=0}^{\infty} \Lambda_{n m}^{(i j)} C_{n}^{(i)^{*}}\left(\mathbf{r}_{1}\right) C_{m}^{(j)}\left(\mathbf{r}_{2}\right) & \text { for } i \neq j .\end{cases}
$$

For a vector-based expansion of $\mathrm{w}\left(\theta_{1}, \phi_{1}, \theta_{2}, \phi_{2}\right)$ in terms of the set of coherent modes $\Psi_{n}(\theta, \phi)$, we similarly find

$$
\mathbb{W}\left(\mathbf{r}_{1}, \mathbf{r}_{2}\right)=\sum_{n=0}^{\infty} \lambda_{n} \mathbf{C}_{n}\left(\mathbf{r}_{1}\right) \mathbf{C}_{n}^{\dagger}\left(\mathbf{r}_{2}\right)
$$

where

$$
\begin{aligned}
\mathbf{C}_{n}\left(\mathbf{r}_{l}\right)= & K \int_{0}^{2 \pi} \int_{0}^{\alpha} \boldsymbol{\Psi}_{n}\left(\theta_{l}, \phi_{l}\right) \exp \left[i k R_{l} \sin \theta_{l} \cos \left(\phi_{l}-\varphi_{l}\right)\right] \\
& \times e^{i k Z \cos \theta_{l}} \sin \theta_{l} \mathrm{~d} \theta_{l} \mathrm{~d} \phi_{l} .
\end{aligned}
$$

Comparing Eqs. (23) and (25) to the definition of the scalar- and vector-based expansions given by Eqs. (23) and (24), respectively, it is apparent that the scalar (vector) coherent modes in the focal region can be found by focusing the coherent modes on the reference sphere by use of the scaled Debye-Wolf integral with scalar (vector) kernel. This result is expected, because by construction the modes are fully spatially and temporally coherent in addition to being statistically independent. Consequently, each coherent mode can be propagated independently using more familiar ideas from coherent optical theories. It should, however, be noted that

$$
\int_{0}^{2 \pi} \int_{0}^{\infty} \mathbf{C}_{n}^{\dagger}\left(\mathbf{r}_{l}\right) \mathbf{C}_{m}\left(\mathbf{r}_{l}\right) R_{l} \mathrm{~d} R_{l} \mathrm{~d} \varphi_{l}=|K|^{2} \delta_{n m},
$$

where $\delta_{n m}$ is the Kronecker delta, meaning that to maintain orthonormality it is necessary to normalize the coherent modes by the factor $|K|$, which yields the alternative, albeit equivalent, expansion

$$
\mathbb{W}\left(\mathbf{r}_{1}, \mathbf{r}_{2}\right)=\sum_{n=0}^{\infty} \lambda_{n}|K|^{2} \hat{\mathbf{C}}_{n}\left(\mathbf{r}_{1}\right) \hat{\mathbf{C}}_{n}^{\dagger}\left(\mathbf{r}_{2}\right),
$$

where $\hat{\mathbf{C}}_{n}(\mathbf{r})$ denotes a renormalized coherent mode.

Finally, we consider coherent mode expansions of the cross-spectral density $\widetilde{\mathbb{W}}\left(\theta_{1}, \phi_{1}, \theta_{2}, \phi_{2}\right)$ in the back focal plane. The scalar- and vector-based coherent modes are denoted $\widetilde{\psi}_{n}^{(i)}(\theta, \phi)$ and $\widetilde{\boldsymbol{\Psi}}_{n}(\theta, \phi)$, respectively. To formulate this problem we need only relate the coherent modes on the reference sphere to those in the back focal plane. For the vector-based expansion Eq. (14) gives $\boldsymbol{\Psi}_{n}(\theta, \phi)$ $=\mathbb{P}(\theta, \phi) \cdot \widetilde{\Psi}_{n}(\theta, \phi)$. When considering the scalar-based expansion, however, the mixing of the elements of the CSDM caused by the transformation of Eq. (14) means that the focused CSDM cannot be expressed in the form of Eq. (23). The lack of a simple, analytic correspondence between the coherent modes in the back focal plane and those in the focal region hence suggests that a scalarbased coherent mode expansion is unsuitable for focusing in electromagnetic problems. Consequently, we shall consider only vector-based expansions in the derivations of Section 5.

At this juncture it is convenient to define a number of different metrics that are commonly used to describe partially coherent light. There is again much dispute regarding the appropriateness and meaning of these quantities; however, here we refrain from such discussions but in- 
stead examine the implications of focusing in terms of each metric. In particular, we consider the degree of spectral coherence defined in [32] as

$$
\eta^{2}\left(\mathbf{r}_{1}, \mathbf{r}_{2}\right)=\frac{\operatorname{tr}\left[\mathbb{W}\left(\mathbf{r}_{1}, \mathbf{r}_{2}\right)\right]^{2}}{\operatorname{tr}\left[\mathbb{W}\left(\mathbf{r}_{1}, \mathbf{r}_{1}\right)\right] \operatorname{tr}\left[\mathbb{W}\left(\mathbf{r}_{2}, \mathbf{r}_{2}\right)\right]}
$$

and the degree of spectral coherence defined in [31] as

$$
\zeta^{2}\left(\mathbf{r}_{1}, \mathbf{r}_{2}\right)=\frac{\left\|\mathbb{W}\left(\mathbf{r}_{1}, \mathbf{r}_{2}\right)\right\|_{F}^{2}}{\operatorname{tr}\left[\mathbb{W}\left(\mathbf{r}_{1}, \mathbf{r}_{1}\right)\right] \operatorname{tr}\left[\mathbb{W}\left(\mathbf{r}_{2}, \mathbf{r}_{2}\right)\right]}
$$

where $\operatorname{tr}[\cdots]$ and $\|\cdots\|_{F}$ denote the matrix trace and the Frobenius norm respectively. Analogous definitions hold for the light before focusing. Since the CSDM will in general change upon focusing, then so too will the associated degrees of spectral coherence $\eta$ and $\zeta$. Numerical examples of this will be given in Section 6; however, it is informative to consider the effective degree of coherence, $\bar{\zeta}$, over the domain $D$ for a general CSDM, as defined in [31] by

$$
\bar{\zeta}^{2}=\frac{\int_{D} \int_{D}\left\|\mathbb{W}\left(\mathbf{r}_{1}, \mathbf{r}_{2}\right)\right\|_{F}^{2} \mathrm{~d} \mathbf{r}_{1} \mathrm{~d} \mathbf{r}_{2}}{\int_{D} \operatorname{tr}\left[\mathbb{W}\left(\mathbf{r}_{1}, \mathbf{r}_{1}\right)\right] \mathrm{d} \mathbf{r}_{1} \int_{D} \operatorname{tr}\left[\mathbb{W}\left(\mathbf{r}_{2}, \mathbf{r}_{2}\right)\right] \mathrm{d} \mathbf{r}_{2}}
$$

Before and after focusing, $\bar{\zeta}^{2}$ evaluates to $\sum_{n=0}^{\infty} \lambda_{n}^{2} /\left[\sum_{n=0}^{\infty} \lambda_{n}\right]^{2}$, and it is therefore possible to conclude that the effective degree of spectral coherence $\bar{\zeta}$ is unchanged upon focusing. Unfortunately, no conservation rule holds for $\bar{\eta}^{2}$ that could be defined in an analogous way.

\section{HARMONIC ANGULAR DEPENDENCE}

Further simplifications of the focusing integrals of Eqs. (10) and (15) can be made if certain symmetry conditions hold. In particular we shall analytically evaluate the azimuthal integration when the coherent modes (on either the reference sphere or the back focal plane) have a harmonic angular dependence, i.e., $\boldsymbol{\Psi}_{n}(\theta, \phi)=\boldsymbol{\Psi}_{n}(\theta) \sin m \phi$ or $\boldsymbol{\Psi}_{n}(\theta) \cos m \phi$ and similarly for $\widetilde{\boldsymbol{\Psi}}_{n}(\theta, \phi)$, where $m \in \mathbb{Z}_{0}^{+}$.

To consider the assertion of harmonic angular dependence on the reference sphere, it is sufficient to consider the $\mathbf{C}_{n}\left(\mathbf{r}_{l}\right)$ integrals of Eq. (25) such that

$$
\begin{aligned}
\mathbf{C}_{n}^{I}\left(\mathbf{r}_{l}\right)= & K \int_{0}^{2 \pi} \int_{0}^{\alpha} \boldsymbol{\Psi}_{n}\left(\theta_{l}\right)\left\{\begin{array}{c}
\sin m \phi_{l} \\
\cos m \phi_{l}
\end{array}\right\} \exp \left[i k R_{l} \sin \theta_{l}\right. \\
& \left.\times \cos \left(\phi_{l}-\varphi_{l}\right)\right] e^{i k Z \cos \theta_{l}} \sin \theta_{l} \mathrm{~d} \theta_{l} \mathrm{~d} \phi_{l} .
\end{aligned}
$$

Employing the well-known identity [33]

$$
\int_{0}^{2 \pi}\left\{\begin{array}{c}
\sin m \alpha \\
\cos m \alpha
\end{array}\right\} \exp [i a \cos (\alpha-\beta)] \mathrm{d} \alpha=2 \pi i^{m}\left\{\begin{array}{c}
\sin m \beta \\
\cos m \beta
\end{array}\right\} J_{m}(a),
$$

where $J_{m}(a)$ is the Bessel function of the first kind of order $m$, yields

$$
\begin{aligned}
\mathbf{C}_{n}^{I}\left(\mathbf{r}_{l}\right)= & 2 \pi i^{m} K\left\{\begin{array}{l}
\sin m \varphi_{l} \\
\cos m \varphi_{l}
\end{array}\right\} \\
& \times \int_{0}^{\alpha} \boldsymbol{\Psi}_{n}\left(\theta_{l}\right) J_{m}\left(k R_{l} \sin \theta_{l}\right) e^{i k Z \cos \theta_{l}} \sin \theta_{l} \mathrm{~d} \theta_{l} .
\end{aligned}
$$

Alternatively, when considering coherent modes on the back focal plane we have

$$
\begin{aligned}
\mathbf{C}_{n}^{I I}\left(\mathbf{r}_{l}\right)= & K \int_{0}^{2 \pi} \int_{0}^{\alpha} \mathbb{P}\left(\theta_{l}, \phi_{l}\right) \tilde{\boldsymbol{\Psi}}_{n}\left(\theta_{l}\right) \\
& \times\left\{\begin{array}{l}
\sin m \phi_{l} \\
\cos m \phi_{l}
\end{array}\right\} e^{i k R_{l} \sin \theta_{l} \cos \left(\phi_{l}-\varphi_{l}\right)} e^{i k Z \cos \theta_{l}} \sin \theta_{l} \mathrm{~d} \theta_{l} \mathrm{~d} \phi_{l} .
\end{aligned}
$$

Expanding $\mathbb{P}(\theta, \phi)$ gives

$$
\mathbb{P}(\theta, \phi)=\frac{a(\theta)}{2}\left(\begin{array}{ccc}
p_{1}+p_{2} \cos 2 \phi & p_{2} \sin 2 \phi & p_{3} \cos \phi \\
p_{2} \sin 2 \phi & p_{1}-p_{2} \cos 2 \phi & p_{3} \sin \phi \\
-p_{3} \cos \phi & -p_{3} \sin \phi & p_{4}
\end{array}\right),
$$

where

$$
\begin{aligned}
& p_{1}=\cos \theta+1, \\
& p_{2}=\cos \theta-1, \\
& p_{3}=2 \sin \theta, \\
& p_{4}=2 \cos \theta .
\end{aligned}
$$

Again using Eq. (32) we can perform the integration over $\phi_{l}$ to give

$$
\mathbf{C}_{n}^{I I}\left(\mathbf{r}_{l}\right)=\frac{K}{2}\left(\begin{array}{c}
\Theta_{2,-m, 2}^{n, x, s}-\Theta_{2,-m, 2}^{n, y, c}-i \Theta_{1,-m, 3}^{n, z, s}+2 \Theta_{0, m, 1}^{n, x, s}+i \Theta_{1, m, 3}^{n, z, s}-\Theta_{2, m, 2}^{n, x, s}+\Theta_{2, m, 2}^{n, y, c} \\
-\Theta_{2,-m, 2}^{n, x, c}-\Theta_{2,-m, 2}^{n, y, s}+i \Theta_{1,-m, 3}^{n, z, c}+2 \Theta_{0, m, 1}^{n, y, s}-i \Theta_{1, m, 3}^{n, z, c}+\Theta_{2, m, 2}^{n, x, c}+\Theta_{2, m, 2}^{n, y, s} \\
i \Theta_{1,-m, 3}^{n, x, s}-i \Theta_{1,-m, 3}^{n, y, c}+2 \Theta_{0, m, 4}^{n, z, s}-i \Theta_{1, m, 3}^{n, x, s}+i \Theta_{1, m, 3}^{n, y, c}
\end{array}\right)
$$

for a sinusoidal angular dependence or 


$$
\mathbf{C}_{n}^{I I}\left(\mathbf{r}_{l}\right)=\frac{K}{2}\left(\begin{array}{c}
-\Theta_{2,-m, 2}^{n, x, c}-\Theta_{2,-m, 2}^{n, y, s}+i \Theta_{1,-m, 3}^{n, z, c}+2 \Theta_{0, m, 1}^{n, x, c}+i \Theta_{1, m, 3}^{n, z, c}-\Theta_{2, m, 2}^{n, x, c}-\Theta_{2, m, 2}^{n, y, s} \\
-\Theta_{2,-m, 2}^{n, x, s}+\Theta_{2,-m, 2}^{n, y, c}+i \Theta_{1,-m, 3}^{n, z, s}+2 \Theta_{0, m, 1}^{n, y, c}+i \Theta_{1, m, 3}^{n, z, s}-\Theta_{2, m, 2}^{n, x s}+\Theta_{2, m, 2}^{n, y, c} \\
-i \Theta_{1,-m, 3}^{n, x, c}-i \Theta_{1,-m, 3}^{n, y, s}+2 \Theta_{0, m, 4}^{n, z, c}-i \Theta_{1, m, 3}^{n, x, c}-i \Theta_{1, m, 3}^{n, y, s}
\end{array}\right)
$$

for a cosinusoidal angular dependence, where

$$
\begin{aligned}
\Theta_{q, \pm m, u}^{n, v, t}\left(\mathbf{r}_{l}\right)= & 2 \pi i^{ \pm m}\left\{\begin{array}{c}
\sin (q \pm m) \varphi_{l} \\
\cos (q \pm m) \varphi_{l}
\end{array}\right\} \int_{0}^{\alpha} a\left(\theta_{l}\right) \tilde{\Psi}_{n}^{v}\left(\theta_{l}\right) p_{u} \\
& \times \sin \theta_{l} J_{q \pm m}\left(k R_{l} \sin \theta_{l}\right) e^{i k Z \cos \theta_{l}} \mathbf{d} \theta_{l},
\end{aligned}
$$

where $\widetilde{\Psi}_{n}^{v}$ denotes the $v$ th component of $\widetilde{\boldsymbol{\Psi}}_{n}$ and the sin (cos) term is taken for $t=s(c)$. Evaluation of the single integrals of Eq. (39) is all that is necessary to calculate the CSDM of focused, inhomogeneous, partially polarized, partially coherent light for which the coherent modes have a harmonic angular dependence.

In coherence calculations the assumption of a circularly symmetric CSDM is often made (whereby either w $\left(\theta_{1}, \phi_{1}, \theta_{2}, \phi_{2}\right)=$ w $\left(\theta_{1}, \theta_{2}\right)$ or $\left.\widetilde{\mathbb{W}}\left(\theta_{1}, \phi_{1}, \theta_{2}, \phi_{2}\right)=\widetilde{\mathbb{W}}\left(\theta_{1}, \theta_{2}\right)\right)$ because it allows the dimensionality of analysis to be reduced. Circularly symmetry in the CSDM is inherited by the coherent modes, and hence this frequently considered scenario is given as a special case $(m=0)$ of the preceding analysis. We have demonstrated that even under less stringent assumptions the dimensionality of the problem can still be reduced. Finally, it should be noted that in the preceding analysis it was assumed that each field component of the vector-based coherent modes had the same harmonic behaviour. This assumption is, however, not required since Eq. (32) can still be used to form a family of integrals similar to that defined by Eq. (39). An example of this type is considered in the next section.

\section{NUMERICAL EXAMPLES}

\section{A. Radially Polarized Laguerre-Gauss Modes}

By way of example we consider a beamlike source formed by the superposition of mutually uncorrelated, radially polarized Laguerre-Gauss modes located in the back focal plane of a lens. Radially polarized beams are becoming increasingly popular for two reasons: first, upon focusing they give a focal spot narrower than the Rayleigh diffraction limit [34], and second, Laguerre-Gauss modes are obtained for typical laser cavities with circular geometries. In this scenario the CSDM in the back focal plane is of the form $\widetilde{\mathbb{W}}\left(\theta_{1}, \phi_{1}, \theta_{2}, \phi_{2}\right)$ $=\sum_{n=0}^{\infty} \lambda_{n} \widetilde{\boldsymbol{\Psi}}_{n}\left(\theta_{1}, \phi_{1}\right) \widetilde{\boldsymbol{\Psi}}_{n}^{\dagger}\left(\theta_{2}, \phi_{2}\right)$, where

$$
\widetilde{\Psi}_{n}(\theta, \phi)=\widetilde{\Psi}_{n}(\theta)\left(\begin{array}{c}
\cos \phi \\
\sin \phi \\
0
\end{array}\right)
$$

and

$$
\widetilde{\Psi}_{n}(\theta)=\left(\frac{2}{\pi \mu^{2}}\right)^{1 / 2} L_{n}\left(\frac{2 \sin ^{2} \theta}{\mu^{2}}\right) \exp \left(-\frac{\sin ^{2} \theta}{\mu^{2}}\right) .
$$

$L_{n}$ represents the $n$ th-order Laguerre polynomial and $\mu$ is a frequency-dependent parameter. We further consider the case discussed in [35] for which $\lambda_{n}=\pi\left(1-q^{2}\right) q^{2 n} / 2 \mu^{2}$ for $0<q<1$. The parameter $\mu$ is a measure of the beam diameter measured in focal lengths, while $q$ determines the effective degree of spectral coherence via $\bar{\zeta}^{2}=(1$ $\left.-q^{2}\right) /\left(1+q^{2}\right)$, with the limits $q \rightarrow 0(q \rightarrow 1)$ giving a fully spatially (un)correlated source. The beam diameter as specified by $\mu$ will be held constant throughout the remainder of this work to avoid extraneous effects resulting from a different apodization of the beam.

Following the analysis given in Section 5, the focused coherent modes are found to be

$$
\mathbf{C}_{n}^{I I}\left(\mathbf{r}_{l}\right)=K\left(\begin{array}{c}
\left(\Theta_{0,1,1}^{n}-\Theta_{2,-1,2}^{n}\right) \cos \varphi \\
\left(\Theta_{0,1,1}^{n}-\Theta_{2,-1,2}^{n}\right) \sin \varphi \\
-i \Theta_{1,-1,3}^{n}
\end{array}\right)
$$

where

$$
\begin{aligned}
\Theta_{0,1,1}^{n}\left(\mathbf{r}_{l}\right)= & 2 \pi i \int_{0}^{\alpha} a\left(\theta_{l}\right) \widetilde{\Psi}_{n}\left(\theta_{l}\right)\left(\cos \theta_{l}+1\right) \\
& \times \sin \theta_{l} J_{1}\left(k R_{l} \sin \theta_{l}\right) e^{i k Z \cos \theta_{l}} \mathrm{~d} \theta_{l},
\end{aligned}
$$

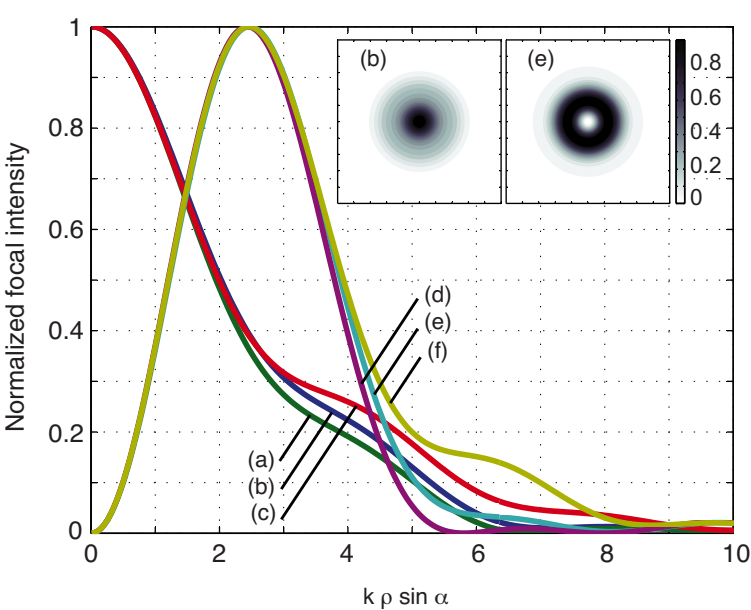

Fig. 2. (Color online) Radial line scans $(\varphi=0)$ and full transverse focused intensity distributions for a radially polarized beam source for (a) $\bar{\zeta}=0$ ( $q=0$, coherent), (b) $\bar{\zeta}=1 / 3 \quad(q=0.62)$, and (c) $\bar{\zeta}=2 / 3(q=0.89)$. (d), (e), (f) Similar line scans for an azimuthally polarized beam. For numerical calculations we assumed an aplanatic lens of numerical aperture 0.97. Furthermore, the values $\mu=1$ and $\lambda=405 \mathrm{~nm}$ were used. Note that peak intensity has been normalized to unity in all cases for easy comparison. 


$$
\begin{aligned}
\Theta_{2,-1,2}^{n}\left(\mathbf{r}_{l}\right)= & -2 \pi i \int_{0}^{\alpha} a\left(\theta_{l}\right) \widetilde{\Psi}_{n}\left(\theta_{l}\right)\left(\cos \theta_{l}-1\right) \\
& \times \sin \theta_{l} J_{1}\left(k R_{l} \sin \theta_{l}\right) e^{i k Z \cos \theta_{l}} \mathrm{~d} \theta_{l}, \\
\Theta_{1,-1,3}^{n}\left(\mathbf{r}_{l}\right)= & -4 \pi i \int_{0}^{\alpha} a\left(\theta_{l}\right) \widetilde{\Psi}_{n}\left(\theta_{l}\right) \\
& \times \sin ^{2} \theta_{l} J_{0}\left(k R_{l} \sin \theta_{l}\right) e^{i k Z \cos \theta_{l}} \mathrm{~d} \theta_{l} .
\end{aligned}
$$

Using these coherent modes it is possible to calculate the focal intensity distribution for sources of differing effective degree of spectral coherence $\bar{\zeta}$. In Fig. 2 we have plotted transverse line scans $(\varphi=0, Z=0)$ for sources with $\bar{\zeta}=0,1 / 3$ and $2 / 3$. Although there is little effect on the width of the transverse profile, we note with reference to Fig. 3 that there is a modest extension in the depth of field as the source becomes more incoherent. There is also a slight increase in the energy density in the wings of the transverse profile. We note that due to the apodization over the pupil, the focal spot is broader than that for uniform intensity since the contribution from the longitudinal field component, responsible for the narrow spot for the unapodized case, is reduced.

Figures 4(a) and 4(c) show plots of the degrees of spectral coherence, $\eta$ and $\zeta$, between points located along the positive $x$ axis $\left(\phi_{1}=\phi_{2}=0\right)$ in the focal plane. Unity degree of coherence between two points implies that were the field from these points brought together, the resulting interference fringes would have a visibility of unity. Consequently if $\mathbf{r}_{1}=\mathbf{r}_{2}$ (dashed line) then $\eta$ automatically evaluates to unity, as can be seen in Fig. 4(a). However, this is not in general true for the $\zeta$, since this also measures the correlations between individual components of the electric field. The rotation of the electric field vector by a lens can introduce differing stochastic behavior in orthogonal field components, hence resulting in the possibility of $\zeta(\mathbf{r}, \mathbf{r}) \neq 1$ as can be seen along the diagonal in Fig. 4 . The differences between the two metrics are more fully discussed in [32].
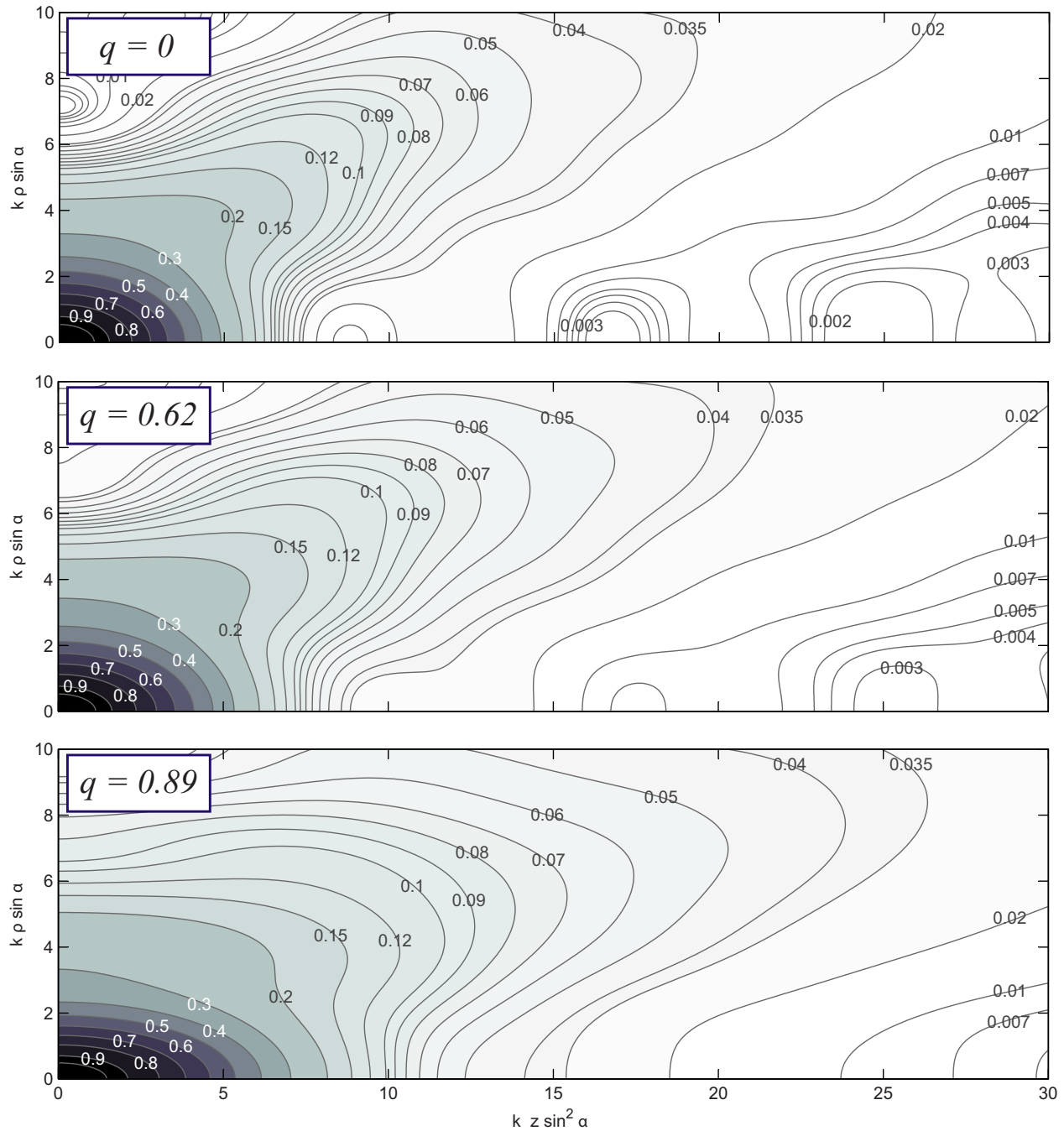

Fig. 3. (Color online) Normalized axial focal intensity distributions $(\varphi=0)$ for partially coherent radially polarized collimated sources with differing effective degrees of spectral coherence as specified by $\bar{\zeta}=0$ (top), $\bar{\zeta}=1 / 3$ (center) and $\bar{\zeta}=2 / 3$ (bottom). Other simulation parameters used were the same as in Fig. 2. 

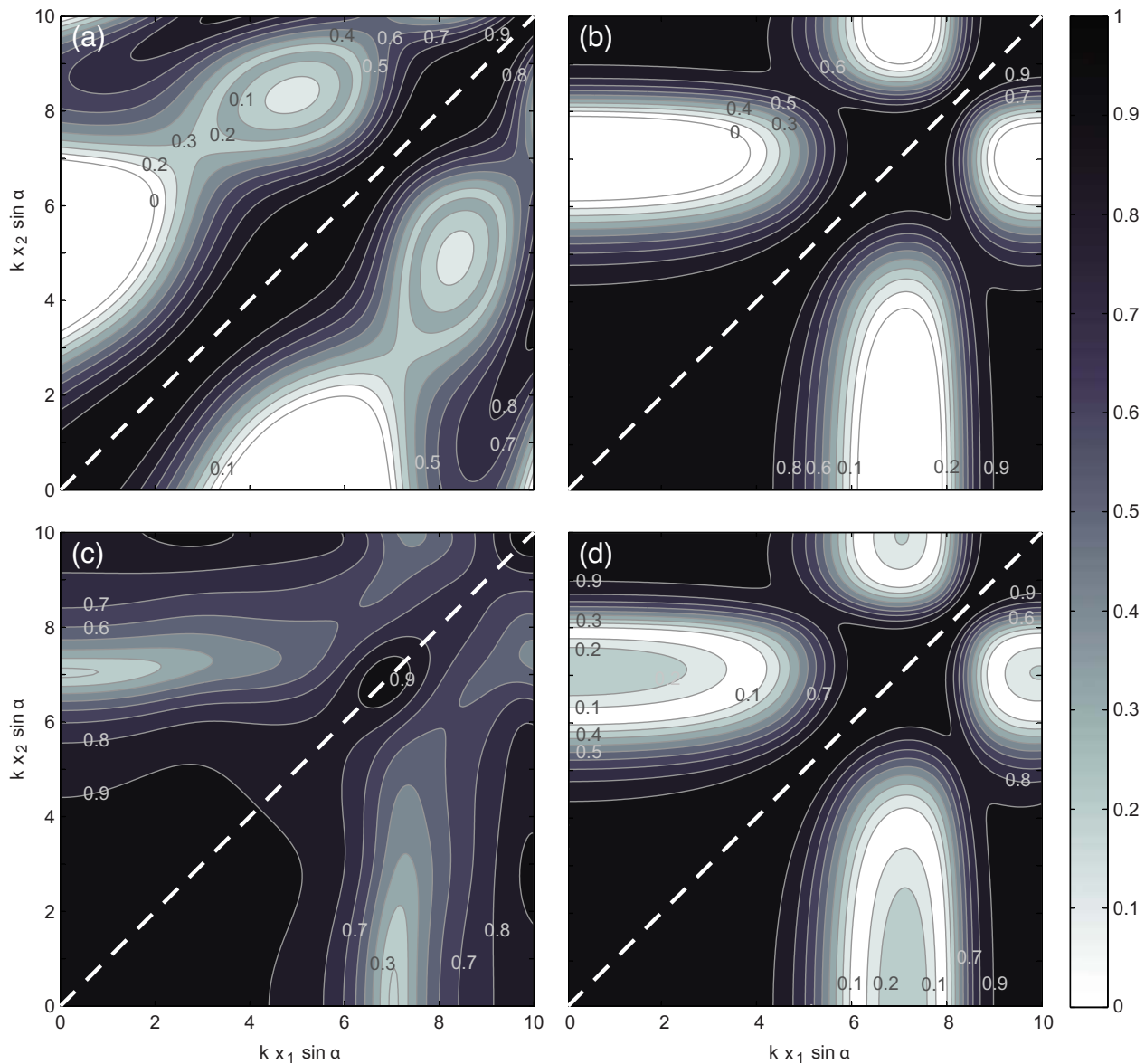

Fig. 4. (Color online) Plots of the degrees of coherence, $|\eta|$ (top) and $\zeta$ (bottom) between two points $x_{1}$ and $x_{2}$ on the positive $x$ axis in the focal plane for focused partially coherent radially (left) and azimuthally (right) polarized collimated light. A value of $q=0.62$ was used, while other simulation parameters were the same as in Fig. 2.

\section{B. Azimuthally Polarized Laguerre-Gauss Modes}

Azimuthally polarized beams are equally seeing attention in the literature and upon focusing produce a focal ring useful, for example, in STED microscopy [36]. In this case the vectorial coherent modes are of the form

$$
\widetilde{\boldsymbol{\Psi}}_{n}(\theta, \phi)=\widetilde{\Psi}_{n}(\theta)\left(\begin{array}{c}
\sin \phi \\
-\cos \phi \\
0
\end{array}\right),
$$

which in the focal region yields

$$
\mathbf{C}_{n}^{I I}\left(\mathbf{r}_{l}\right)=K\left(\begin{array}{c}
\left(\Theta_{0,1,1}^{n}+\Theta_{2,-1,2}^{n}\right) \sin \varphi \\
-\left(\Theta_{0,1,1}^{n}+\Theta_{2,-1,2}^{n}\right) \cos \varphi \\
0
\end{array}\right) .
$$

Again, transverse line scans of the focal intensity distribution are shown in Fig. 2, while the axial intensity distribution is shown in Fig. 5. Conclusions similar to those made for the radially polarized source can be drawn for an azimuthally polarized source; however, the augmentation of the wings of the transverse intensity profile is more pronounced.

Figures 4(b) and 4(d) again show plots of the degrees of spectral coherence, $\eta$ and $\zeta$, between points located along the positive $x$ axis in the focal plane. For azimuthally po- larized illumination the resulting plots are very similar, because along the $x$ axis the focused coherent modes are purely $y$ polarized.

\section{CONCLUSIONS}

This work was undertaken with a view to developing a general description of focusing of partially polarized, partially coherent electromagnetic waves capable of handling spatially inhomogeneous statistical properties across the pupil of the focusing lens(es). This objective has been achieved by use of the scaled Debye-Wolf diffraction integral, which places few constraints on the system geometry since it is valid for high numerical aperture lenses of arbitrary Fresnel number. Furthermore, we have shown that by employing a coherent mode representation of the CSDM it is possible to reduce the four-dimensional integrals to two-dimensional ones. This allows substantial computational gains to be made. Analysis of the focusing operation has been performed in terms of the CSDM across both the Gaussian reference sphere and the back focal plane in terms of scalar- and vector-based coherent modes, since both are frequently used in optical calculations. It was found, however, that due to mixing of different components of the electric field that occurs in highnumerical-aperture optical systems, scalar-based coherent mode expansions can be unsuitable. We were 

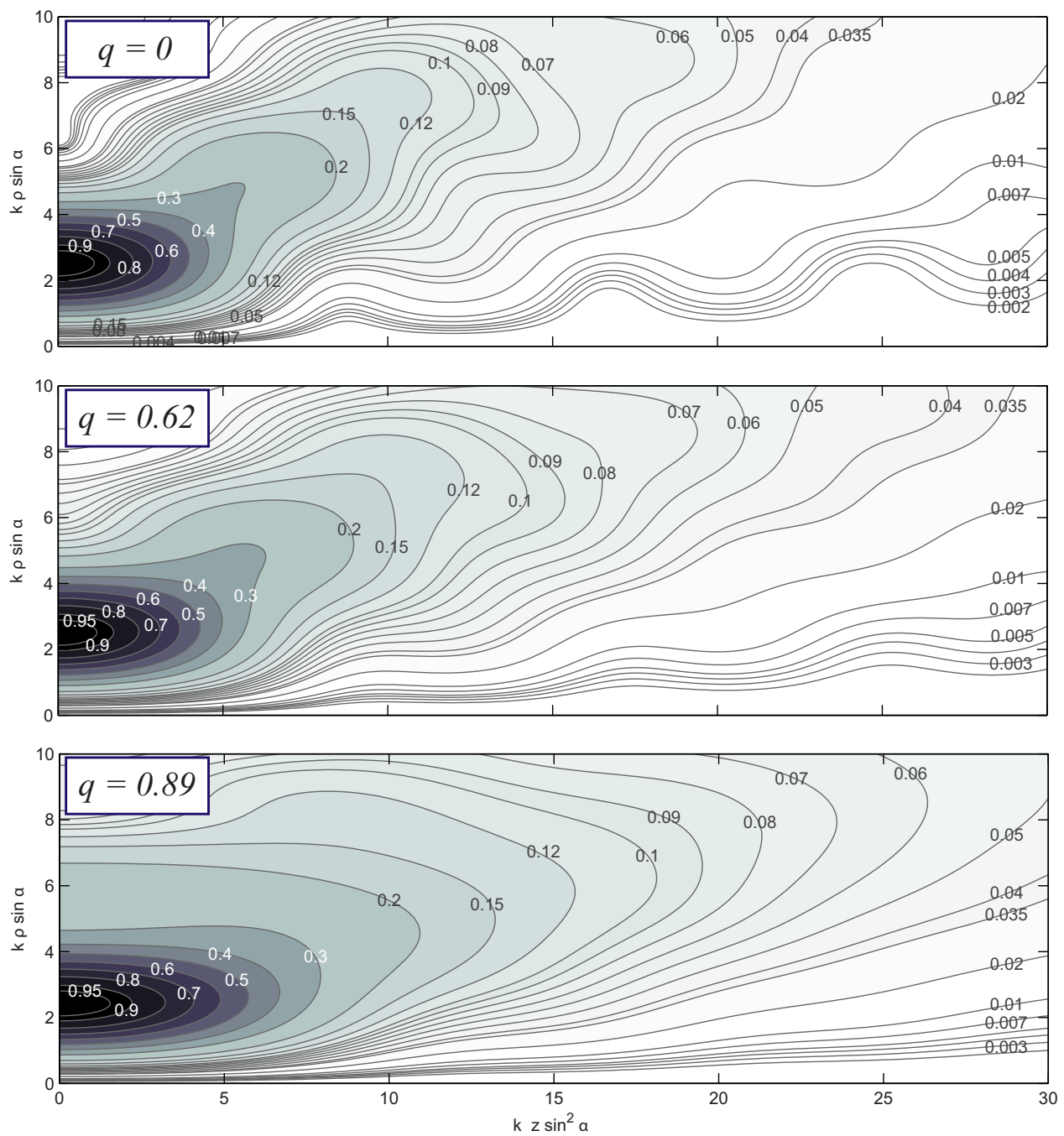

Fig. 5. (Color online) Normalized axial focal intensity distributions in the same manner as Fig. 3 for partially coherent azimuthally polarized collimated sources with differing effective degrees of spectral coherence.

also able to show that the effective degree of spectral coherence $\bar{\zeta}$ of an electromagnetic beam is unchanged upon focusing.

In addition, although the imposition of circular symmetry is often made in the analysis of optical systems to make calculations more mathematically tractable and to reduce the dimensionality of the problem, we have shown that these benefits are still realizable with the less stringent requirement of a harmonic angular dependence of the coherent modes. A couple of examples were also briefly discussed to highlight our mathematical method.

As a final comment we are mindful that we have concentrated solely on the second-order statistical properties as encapsulated by the CSDM. That said, it is in principle possible to extend Eqs. (10) and (15) to calculate higherorder statistical moments of focused light. Since knowledge of all the moments of a random process, provides a full description of the process, it is thus possible to fully account for the effect of focusing on randomly fluctuating electromagnetic waves within the framework discussed.

\section{ACKNOWLEDGMENTS}

We acknowledge the financial support of the Engineering and Physical Sciences Research Council (EPSRC), UK, and the European Union via the SURPASS project, grant no. 224226 , without which this work would not have been possible.

\section{REFERENCES}

1. J. J. Stamnes, Waves in Focal Regions (Adam Hilger, 1986).

2. V. S. Ignatowsky, "Diffraction by a lens of arbitrary aperture," Trans. Opt. Inst. Pet. 1, 1-36 (1919).

3. V. S. Ignatowsky, "The relationship between geometrical and wave optics and diffraction of homocentrical beams," Trans. Opt. Inst. Pet. 1, 1-30 (1920).

4. E. Wolf, "Electromagnetic diffraction in optical systems I. An integral representation of the image field," Proc. R. Soc. London, Ser. A 253, 349-357 (1959).

5. B. Richards and E. Wolf, "Electromagnetic diffraction in optical systems II. Structure of the image field in an aplanatic system," Proc. R. Soc. London, Ser. A 253, 358-379 (1959).

6. W. Wang, A. Friberg, and E. Wolf, "Focusing of partially coherent light in systems of large Fresnel numbers," J. Opt. Soc. Am. A 14, 491-496 (1997).

7. D. Fischer and T. Visser, "Spatial correlation properties of focused partially coherent light," J. Opt. Soc. Am. A 21, 2097-2102 (2004).

8. C. Rydberg, "First- and second-order statistics of partially coherent, high-numerical-aperture optical fields," Opt. Lett. 33, 104-106 (2008). 
9. X. Liu and J. Pu, "Focal shift and focal switch of partially coherent light in dual-focus systems," Opt. Commun. 252, 262-267 (2005).

10. K. Lindfors, T. Setälä, M. Kaivola, and A. Friberg, "Degree of polarization in tightly focused optical fields," J. Opt. Soc. Am. A 22, 561-568 (2005).

11. Z. Zhang, J. Pu, and X. Wang, "Focusing of partially coherent Bessel-Gaussian beams through a highnumerical-aperture objective," Opt. Lett. 33, 49-51 (2008).

12. T. van Dijk, G. Gbur, and T. Visser, "Shaping the focal intensity distribution using spatial coherence," J. Opt. Soc. Am. A 25, 575-581 (2008).

13. R. H. Lehmberg, A. J. Schmitt, and S. E. Bodner, "Theory of induced spatial incoherence," J. Appl. Phys. 62, 2680-02701 (1987).

14. Y. Kato, K. Mima, N. Miyanaga, S. Arinaga, Y. Kitagawa, M. Nakatsuka, and C. Yamanaka, "Random phasing of high-power lasers for uniform target acceleration and plasma-instability suppression," Phys. Rev. Lett. 53, 1057-1060 (1984).

15. W. Gao, "Effects of coherence and vector properties of the light on the resolution limit in stimulated emission depletion fluorescence microscopy,” J. Opt. Soc. Am. A 25, 1378-1382 (2008).

16. P. Török, "Focusing of electromagnetic waves through a dielectric interface by lenses of finite Fresnel number," J. Opt. Soc. Am. A 15, 3009-3015 (1998).

17. J. W. Goodman, Statistical Optics (Wiley, 2000).

18. N. Wiener, "Coherency matrices and quantum theory," J. Math. Phys. 7, 109-125 (1928).

19. N. Wiener, "Harmonic analysis and the quantum theory," J. Franklin Inst. 207, 525-553 (1929).

20. E. Wolf, "Coherence properties of partially polarized electromagnetic radiation," Nuovo Cimento 13, 1165-1181 (1959).

21. E. Wolf, Introduction to the Theory of Coherence and Polarization of Light (Cambridge Univ. Press, 2007).

22. P. Török, P. Varga, Z. Laczik, and G. Booker, "Electromagnetic diffraction of light focused through a planar interface between materials of mismatched refractive indices: an integral representation," J. Opt. Soc. Am. A 12, 325-332 (1995).

23. P. Török, P. D. Higdon, and T. Wilson, "On the general properties of polarised light conventional and confocal microscopes," Opt. Commun. 148, 300-315 (1998).

24. D. J. Innes and A. L. Bloom, "Design of optical systems for use with laser beams," Spectra-Phys. Laser Tech. Bull. 5, $1-10(1966)$

25. E. Wolf, "New theory of partial coherence in the spacefrequency domain. Part I: spectra and cross spectra of steady-state sources," J. Opt. Soc. Am. 72, 343-351 (1982).

26. C. Palma and G. Cincotti, "Imaging of $J_{0}$ correlated BesselGauss beams," IEEE J. Quantum Electron. 33, 1032-1040 (1997).

27. S. Flewett, H. Quiney, C. Tran, and K. Nugent, "Extracting coherent modes from partially coherent wavefields," Opt. Lett. 34, 2198-2200 (2009).

28. K. Karhunen, "Über lineare Methoden in der Wahrscheinlichkeitsrechnung," Ann. Acad. Sci. Fenn., Ser. A1: Math.-Phys. A137, 1-79 (1947).

29. M. Loève, Probability Theory, 4th ed. (Springer-Verlag, 1978).

30. F. Gori, M. Santarsiero, R. Simon, G. Piquero, R. Borghi, and G. Guattari, "Coherent-mode decomposition of partially polarized, partially coherent sources," J. Opt. Soc. Am. A 20, 78-84 (2003)

31. J. Tervo, T. Setälä, and A. Friberg, "Theory of partially coherent electromagnetic fields in the space-frequency domain,” J. Opt. Soc. Am. A 21, 2205-2215 (2004).

32. D. P. Brown, A. K. Spilman, T. G. Brown, R. Borghi, S. N. Volkov, and E. Wolf, "Spatial coherence properties of azimuthally polarized laser modes," Opt. Commun. 281, 5287-5290 (2008)

33. G. N. Watson, A Treatise on the Theory of Bessel Functions, 2nd ed. (Cambridge Univ. Press, 1995).

34. R. Dorn, S. Quabis and G. Leuchs, "Sharper focus for a radially polarized light beam," Phys. Rev. Lett. 91, 233901 (2003).

35. G. Guattari, C. Palma, and C. Padovani, "Cross-spectral densities with axial symmetry," Opt. Commun. 73, 173-178 (1989).

36. P. Török and P. Munro, "The use of Gauss-Laguerre vector beams in STED microscopy," Opt. Express 12, 3605-3617 (2004). 